\title{
Cytoplasmic line effects for birth weight and preweaning growth traits in the Asturiana de los Valles beef cattle breed
}

\author{
A. Pun ${ }^{\text {a }}$, F. Goyache ${ }^{\mathrm{b}}$, I. Cervantes ${ }^{\text {a }}$, J.P. Gutiérrez ${ }^{\mathrm{a}, *}$ \\ a Departamento de Producción Animal, Universidad Complutense de Madrid, Avda. Puerta de Hierro s/n, E-28040-Madrid, Spain \\ b SERIDA-Somió, C/ Camino de los Claveles 604, E-33203 Gijón (Asturias), Spain
}

\section{A R T I C L E I N F O}

\section{Article history:}

Received 28 June 2011

Received in revised form 9 September 2011

Accepted 12 September 2011

\section{Keywords:}

Average daily gain

Beef cattle

Birth weight

Cytoplasmic effect

Weaning weight

\begin{abstract}
A B S T R A C T
A total of 15,645 records of birth weight (BrW), weaning weight (WW) and average daily gain (ADG) from 6055 cows belonging to 2121 different dam lines were analyzed to quantify the contribution of cytoplasmic line (1) effects to phenotypic variance of preweaning growth traits in a sample of Asturiana de los Valles beef cattle breed. Only dam lines with 2 or more cows having performance records in the database were used. Bayesian estimates were obtained fitting eight different univariate and multivariate models defined depending on the inclusion or not of the permanent maternal environment (c) and the l effects. Univariate models that included both the $\mathrm{c}$ and the 1 effects had the best fit with data (assessed by computing the logarithm of the conditional predictive ordinate; $\log \mathrm{CPO}$ ) for BrW and ADG while for WW this was the case for the model that only included the $c$ effect. For multivariate models, the best fit with data was obtained for the model that only included the $c$ effect $(\log \mathrm{CPO}=$ $-245,899$ ) while the second "better" model was that which only include the 1 effect value $(\log \mathrm{CPO}=-241,108)$. In general, whatever the estimation model used, estimates of heritability for the direct $\left(\mathrm{h}^{2}\right)$ and maternal $\left(\mathrm{m}^{2}\right)$ genetic effects and the genetic correlation between them $\left(r_{a m}\right)$ obtained in the current study for BrW were slightly higher ( $r_{a m}$ more negative) than the most recent estimates reported in the breed. However, this is not true for WW and ADG when multivariate estimation models include the l effect. In these cases, estimates of $h^{2}$ and $\mathrm{m}^{2}$ for WW and ADG tend to be lower and $\mathrm{r}_{\mathrm{am}}$ less negative than those previously estimated. In conclusion, the cytoplasmic line may have a marginal effect on growth performance in beef cattle but not sufficient in magnitude to justify including the leffect in models in beef improvement schemes.
\end{abstract}

(c) 2011 Elsevier B.V. All rights reserved.

\section{Introduction}

Maternal effects include any influence from a dam on its offspring, excluding the effects of directly transmitted genes that affect performance of the offspring (Legates, 1972). Biological mechanisms explaining maternal effects may be partially related to mitochondrial DNA, which is almost exclusively maternally inherited (Gibson et al., 1997).

\footnotetext{
* Corresponding author at: Department of Animal Production, Faculty of Veterinary, UCM. Avda. Puerta del Hierro s/n, E-28040 Madrid, Spain. Tel./ fax: + 34913943767.

E-mail address: gutgar@vet.ucm.es (J.P. Gutiérrez).
}

In dairy cattle, cytoplasmic line (1) effects could explain from 0 to $5 \%$ of variation in performance, with the weight of evidence pointing to the low end of this range (Gibson et al., 1997). In beef cattle, 1 effect has not been identified as a significant source of variation for performance (Northcutt et al., 1991; Tess and MacNeil, 1994; Tess and Robison, 1990) or bull fertility traits (Garmyn et al., 2010). In sheep, 1 effects have been suggested to be of little importance for growth traits in both pure (Maniatis and Pollott, 2002; Snowder et al., 2004.) and composite breeds (Hanford et al., 2003; Van Vleck et al., 2002).

Estimates of 1 effect may be biased downwards due to incomplete or incorrect pedigree information. Misidentification 
of maternal lineages leads to underestimation of betweencytoplasmic line variance (Gibson et al., 1997; Roughsedge et al., 2001). Also, poor data structures make it difficult to differentiate between 1 effects and preferential treatment given to particular dam lineages or the permanent environmental effect associated with a given cow within a line. This is of particular importance in beef cattle due to the existence of genetic and nongenetic relationships between direct and maternal effects affecting performance (see Quintanilla et al., 1999, for a review).

The aim of this research was to quantify the contribution of 1 effects to phenotypic variance of preweaning growth traits in a sample of Asturiana de los Valles beef cattle breed and to ascertain its influence on additive direct and maternal genetic effects associated with these traits.

\section{Materials and methods}

Asturiana de los Valles is a beef cattle breed mainly exploited under semi-extensive or extensive conditions in the wet Cantabrian range area of Northern Spain. Brief descriptions of the management systems and environmental conditions can be found elsewhere (Goyache and Gutiérrez, 2001; Gutiérrez and Goyache, 2002; Gutiérrez et al., 2007).

Production data and pedigree information were obtained from an existing performance recording database (the CORECA database) implemented by the Regional Government of the Principado de Asturias (Northern Spain), through the Asturiana de los Valles Breeders Association (ASEAVA). Due to the small size of the farms, performance recording was implemented on nucleus farms, grouping farms according to their proximity and their production system, further considering the nucleus as the management unit (Cervantes et al., 2010; Goyache et al., 2003a, 2003b, 2005). Animals with identification errors or ambiguous birth dates were excluded from the analysis. Contemporary groups were defined based on nucleus-year of calving.

Following Gutiérrez et al. (2006), production data included only single calving records. Only records with both birth weight (BrW) and weaning weight (WW) were obtained. Age of calf at weaning (AGE) of the available records ranged from 90 to 270 days. Preweaning average daily gain (ADG) was simply computed as ADG $=($ WW-BW $) /$ AGE.

Dam lines were defined in terms of maternal ancestors as unbroken descent through female animals only from an ancestor to a descendant. Only dam lines with 2 or more cows having offspring with performance records in the database were used. The number of cows per dam line ranged from 2 to 15 . The edited dataset contained 15,645 records of $\mathrm{BrW}$, WW and ADG from 6055 cows and 2121 dam lines. The structure of the analysed records is summarised in Table 1. The analysed database included a total of 22,852 animals, of which 1415 were sires and 6949 dams. A total of 1000 sires had progeny in the database.

Genetic parameters together with their standard deviation (SD) were estimated via a multitrait procedure applied to mixed linear models. All runs were carried out using the TM program (Legarra, 2008). The fitted models included the following fixed effects: herd-year of calving as contemporary group (482 levels), calving season (2 levels: from January 1 to June 30 and from July 1 to December 31 ), sex of calf (male or
Table 1

Structure of data used for estimation of genetic parameters for birth weight $(\mathrm{BrW})$, and weaning weight (WW) and preweaning average daily gain (ADG).

\begin{tabular}{lr}
\hline Animals & 22,852 \\
Animals with records & 15,645 \\
Sires with progeny in data & 1000 \\
Cows with progeny in data & 6055 \\
Sires with record and offspring & 107 \\
Cows with record and offspring & 1043 \\
Sire-offspring pairs & 2435 \\
Dam-offspring pairs & 2171 \\
Herd-year of calving (levels) & 482 \\
Calving season (levels) & 2 \\
Calf sex (levels) & 2 \\
Creep feeding (levels) ${ }^{1}$ & 2 \\
Age of cow at calving (linear covariate; levels) & 1 \\
Age of calf at weaning (linear covariate; levels) & 1 \\
Mean \pm s.d. for BrW (kg) & $40 \pm 7.2$ \\
Mean \pm s.d. for WW (kg) & $221.0 \pm 57.7$ \\
Mean \pm s.d. for ADG (g) & $943.8 \pm 243.6$ \\
\hline 1 Effect not included in the models fitted for analyses of BrW.
\end{tabular}

female), and age of the dam at calving, in days, as a linear covariate. The models fitted for analysis of WW and ADG also included the effect of creep feeding ( 2 levels: creep and noncreep) and AGE, in days, as a linear covariate. Regarding random effects, four different models were defined:

Model 1: A univariate animal model including, for each analyzed trait, the additive genetic effect (a), the maternal genetic effect $(\mathbf{m})$ and the covariance between them $\left(\sigma_{\mathrm{am}}\right)$, besides the residual $(\mathbf{e})$.

Model 2: Model 1, but also including the permanent environment associated with dam (c) as a random environmental effect.

Model 3: Model 1, but also including cytoplasmic line (1) effect as a random environmental effect.

Model 4: Model 1, but also including the permanent environment associated with dam (c) and cytoplasmic line (1) effects as random environmental effects.

Model 5: A multivariate animal model including, for each analyzed trait, the additive genetic effect (a), the maternal genetic effect $(\mathbf{m})$, the covariance between them and the covariance between either direct or maternal genetic effects across traits. All of these are dependent on the relationship matrix and the residual (e).

Model 6: Model 5, but also including the permanent environment associated with dam $(\mathbf{c})$ as a random environmental effect independent of the additive relationship matrix and the covariance between either permanent environmental effects. Model 7: Model 5, but also including cytoplasmic line (1) effect as a random environmental effect independent of the additive relationship matrix and the covariance between either cytoplasmic line effects.

Model 8: Model 5, but also including the permanent environment associated with dam $(\mathbf{c})$ and cytoplasmic line (1) effects as random environmental effects and the covariance between either permanent environmental or cytoplasmic line effects. 
The matricial notation of the sets of mixed model equation to be solved is

$\mathbf{y}=\mathbf{X b}+\mathbf{Z a}+\mathbf{N m}+\mathbf{W c}+\mathbf{Q l}+\mathbf{e}$,

with:

$$
\left(\begin{array}{c}
a \\
m \\
c \\
l \\
e
\end{array}\right) \sim N\left(\left[\begin{array}{l}
0 \\
0 \\
0 \\
0 \\
0
\end{array}\right],\left[\begin{array}{ccccc}
G & C & 0 & 0 & 0 \\
C & M & 0 & 0 & 0 \\
0 & 0 & P & 0 & 0 \\
0 & 0 & 0 & L & 0 \\
0 & 0 & 0 & 0 & R
\end{array}\right]\right)
$$

where $\mathbf{G}=\mathbf{A} \otimes \mathbf{G}_{\mathbf{0}}, \mathbf{M}=\mathbf{A} \otimes \mathbf{M}_{\mathbf{0}}, \mathbf{C}=\mathbf{A} \otimes \mathbf{C}_{\mathbf{0}}, \mathbf{P}=\mathbf{I}_{\mathbf{p}} \otimes \mathbf{P}_{\mathbf{0}}, \mathbf{L}=\mathbf{I}_{\mathbf{1}}$ $\otimes \mathbf{L}_{\mathbf{0}}, \mathbf{R}=\mathbf{I}_{\mathbf{e}} \otimes \mathbf{R}_{\mathbf{0}}, \mathbf{y}$ is the vector of observations, $\mathbf{X}$ the incidence matrix of fixed effects, $\mathbf{Z}$ the incidence matrix of animal additive genetic effects, $\mathbf{N}$ the incidence matrix of maternal genetic effects, $\mathbf{W}$ the incidence matrix of permanent environmental effects associated with dam, $\mathbf{Q}$ the incidence matrix of cytoplasmic line effect, $\mathbf{b}$ the vector of fixed effects, $\mathbf{a}$ the vector of direct animal genetic effects, $\mathbf{m}$ the vector of unknown maternal genetic effects, $\mathbf{p}$ the vector of permanent environmental effects associated with dam, $\mathbf{l}$ the vector of cytoplasmic line effect, $\mathbf{e}$ the vector of residuals, $\mathbf{A}$ is the numerator relationship matrix, $\mathbf{G}_{\mathbf{0}}$ is the covariance matrix for additive genetic effects, $\mathbf{M}_{\mathbf{0}}$ is the covariance matrix for maternal genetic effects, $\mathbf{C}_{\mathbf{0}}$ is the covariance matrix between direct additive and maternal genetic effects, $\mathbf{P}_{\mathbf{0}}$ is the covariance matrix for permanent environmental effects associated with dam, $\mathbf{L}_{\mathbf{0}}$ is the covariance matrix for cytoplasmic effects, $\mathbf{R}_{\mathbf{0}}$ is the covariance residual matrix, and $\otimes$ is the Kronecker product, being $\mathbf{G}_{\mathbf{0}}, \mathbf{M}_{\mathbf{0}}, \mathbf{C}_{\mathbf{0}}, \mathbf{P}_{\mathbf{0}}, \mathbf{L}_{\mathbf{0}}$ and $\mathbf{R}_{\mathbf{0}}$, reduced respectively to $\sigma_{\mathrm{a}}^{2}$, the additive genetic variance, $\sigma_{\mathrm{m}}^{2}$, the maternal genetic variance, $\sigma_{\mathrm{am}}$, the covariance between direct additive and maternal genetic effects, $\sigma_{\mathrm{p}}^{2}$, the permanent environmental variance associated with dam, $\sigma_{1}^{2}$, the cytoplasmic line effect variance and $\sigma_{\mathrm{e}}^{2}$, the residual variance, when univariate models are of concern, $\mathbf{I}_{\mathbf{p}}$ the identity matrix of order equal to the number of dams, $\mathbf{I}_{\mathbf{1}}$ the identity matrix of order equal to the number of cytoplasmic lines, and $\mathbf{I}_{\mathbf{e}}$ the identity matrix of order equal to the number records.

Model comparison was computed by the logarithm of the conditional predictive ordinate (logCPO) (Pettit and Young, 1990; Varona and Sorensen, 2010; Varona et al., 1997) for each observation. CPO is a cross-validated predictive approach i.e., predictive distributions conditioned on the observed data with a single data point deleted. The lower the mean logarithm value (most negative value) the better the fit between the observations and the model.

\section{Results}

In Table 2 are given the estimates obtained for the genetic and environmental effects included in the four univariate models fitted (from Model 1 to 4 ). Since the aim of the current study was to ascertain the effect of environmental factors related with cytoplasmic line on the estimates of preweaning growth traits, comparisons were made with estimates obtained using Model 1.

The inclusion of the maternal permanent environmental effect (Model 2) was more important for BrW $\left(c^{2}=0.076 \pm\right.$ $0.018)$ than for WW $(0.028 \pm 0.014)$ or ADG $(0.029 \pm$
0.015). For the latter traits, the influence of the $\mathbf{c}$ effect can be considered statistically non-significant. Also, the inclusion of the $\mathbf{c}$ effect gave lower estimates of $\mathrm{m}^{2}$ for the three traits analyzed (roughly three quarters of that estimated using Model 1) while $h^{2}$ remained basically the same and $r_{a m}$ tended to increase (16\% for BrW, 18\% WW and 6\% ADG, for those estimated using Model 1).

The estimates of $1^{2}$ obtained using Model 3 were null for $\operatorname{BrW}(0.007 \pm 0.006)$, low for WW $(0.033 \pm 0.013)$ and statistically non-significant for ADG $(0.019 \pm 0.010)$. The inclusion of the $\mathbf{l}$ effect in the estimation model did not affect the estimates of the genetic parameters for BrW. For both WW and ADG the inclusion of the l effect in the model gave a slight decrease of the estimates of $\mathrm{m}^{2}(0.090 \pm 0.032$ for WW and $0.130 \pm 0.037$ for ADG) and more negative estimates of $r_{a m}$, particularly for WW $(-0.429 \pm 0.131)$. In any case, estimates of $\mathrm{h}^{2}$ remained basically the same.

Model 4 confirmed the general scenario described above. The c effect influenced to a higher extent $\mathrm{BrW}\left(\mathrm{c}^{2}=0.089 \pm\right.$ $0.018)$ than WW $(0.045 \pm 0.015)$ or ADG $(0.044 \pm 0.016)$. The estimate of $1^{2}$ for BrW obtained using Model 4 was still non-significant while those estimated for WW $(0.048 \pm$ $0.013)$ and ADG $(0.032 \pm 0.012)$ were similar to the corresponding estimates of $\mathrm{c}^{2}$. In any case, Model 4 gave a substantial decrease of the estimates of $\mathrm{m}^{2}$ for WW and ADG (roughly $57 \%$ of those estimated using Model 1) and more negative estimates of $\mathrm{r}_{\mathrm{am}}(-0.682 \pm 0.136$ for $\mathrm{WW}$ and $-0.640 \pm 0.141$ for ADG).

In the cases of BrW and ADG, the best fit with observations was obtained when the full model (Model 4) was used ( $\log$ CPO values of $-164,465$ and $-89,431$, respectively), whereas Model 2 had the best fit for WW $(\log C P O=-179,305)$. However, difference in $\log \mathrm{CPO}$ between Model 2 and Model $4(\log \mathrm{CPO}=$ $-179,197)$ was very small (Table 2 ).

Table 3 gives the estimates obtained for the genetic and environmental effects included in the four multivariate models fitted (from Model 5 to 8). Differences with univariate models were relatively important. The best fit with data was obtained from Model 6 which did not include the 1 effect $(\log \mathrm{CPO}=-245,899)$. The full model (Model 8) had the "worst" $\log$ CPO value $(-232,804)$. The simplest model (Model 5) and the model including the 1 effect (Model 7) had close $\log$ CPO values $(-241,053$ and $-241,108$, respectively).

With respect to the simplest multivariate model (Model 5 ), the "better" model (Model 6) did not affect $h^{2}$ to a large extent but gave a substantial decrease of the estimates of $\mathrm{m}^{2}$ and $\mathrm{r}_{\mathrm{am}}$ for BrW. However, Model 6 gave a 7\% decrease of the estimates of $h^{2}$ but substantial increases of the estimates of $\mathrm{m}^{2}$ and, especially, $\mathrm{r}_{\mathrm{am}}$ for $\mathrm{WW}$ and ADG. The full multivariate model (Model 8) gave higher estimates of $h^{2}$ and $r_{a m}$ than Model 5 for BrW while these are substantially lower (roughly a half of those estimated using Model 5) for WW. Estimates of $\mathrm{h}^{2}$ and $\mathrm{m}^{2}$ for ADG obtained using Model 8 were also lower than those estimated using Model 5 while $r_{\text {am }}$ was $21 \%$ more negative.

Correlations estimated between either direct or maternal genetic effects of the three analyzed traits are given in Table 4 . All the estimated correlations were statistically significant except for the pair aBrW-mADG (using Model 5). In any case, the significant genetic correlations estimated were of the same sign and similar magnitude except for the pair 
Table 2

Mean and SD (in brackets) of the posterior distribution for the within-trait genetic and environmental parameters included in the four univariate models fitted.

\begin{tabular}{|c|c|c|c|c|c|c|c|}
\hline & $h^{2}$ & $\mathrm{~m}^{2}$ & $\mathrm{r}_{\mathrm{am}}$ & $c^{2}$ & $1^{2}$ & $\sigma^{2}$ & $\log \mathrm{CPO}$ \\
\hline \multicolumn{8}{|c|}{ Birth weight } \\
\hline \multirow[t]{2}{*}{ Model 1} & 0.451 & 0.285 & -0.378 & & & 39.820 & $-161,467$ \\
\hline & $(0.060)$ & $(0.048)$ & $(0.087)$ & & & $(1.646)$ & \\
\hline \multirow[t]{2}{*}{ Model 2} & 0.462 & 0.208 & -0.437 & 0.076 & & 39.496 & $-164,176$ \\
\hline & $(0.057)$ & $(0.042)$ & $(0.082)$ & $(0.018)$ & & $(1.445)$ & \\
\hline \multirow[t]{2}{*}{ Model 3} & 0.451 & 0.283 & -0.394 & & 0.007 & 39.657 & $-161,366$ \\
\hline & $(0.056)$ & $(0.047)$ & $(0.082)$ & & $(0.006)$ & $(1.583)$ & \\
\hline \multirow[t]{2}{*}{ Model 4} & 0.470 & 0.186 & -0.509 & 0.089 & 0.023 & 38.991 & $-164,465^{a}$ \\
\hline & $(0.057)$ & $(0.043)$ & $(0.083)$ & $(0.018)$ & $(0.013)$ & $(1.386)$ & \\
\hline \multicolumn{8}{|c|}{ Weaning weight } \\
\hline \multirow[t]{2}{*}{ Model 1} & 0.392 & 0.105 & -0.281 & & & 1456.527 & $-177,983$ \\
\hline & $(0.052)$ & $(0.033)$ & $(0.133)$ & & & $(49.862)$ & \\
\hline \multirow[t]{2}{*}{ Model 2} & 0.401 & 0.081 & -0.333 & 0.028 & & 1453.687 & $-179,305^{a}$ \\
\hline & $(0.055)$ & $(0.032)$ & $(0.136)$ & $(0.014)$ & & $(46.870)$ & \\
\hline \multirow[t]{2}{*}{ Model 3} & 0.403 & 0.090 & -0.429 & & 0.033 & 1425.266 & $-177,620$ \\
\hline & $(0.054)$ & $(0.032)$ & $(0.131)$ & & $(0.013)$ & (49.396) & \\
\hline \multirow[t]{2}{*}{ Model 4} & 0.416 & 0.061 & -0.682 & 0.045 & 0.048 & 1391.576 & $-179,197$ \\
\hline & $(0.054)$ & $(0.026)$ & $(0.136)$ & $(0.015)$ & $(0.013)$ & $(44.136)$ & \\
\hline \multicolumn{8}{|c|}{ Average daily gain } \\
\hline \multirow[t]{2}{*}{ Model 1} & 0.293 & 0.139 & -0.389 & & & $37,916.483$ & $-88,602$ \\
\hline & $(0.047)$ & $(0.036)$ & $(0.127)$ & & & (1323) & \\
\hline \multirow[t]{2}{*}{ Model 2} & 0.297 & 0.107 & -0.413 & 0.029 & & $38,062.029$ & $-89,320$ \\
\hline & $(0.050)$ & $(0.037)$ & $(0.136)$ & $(0.015)$ & & (1289) & \\
\hline \multirow[t]{2}{*}{ Model 3} & 0.303 & 0.130 & -0.472 & & 0.019 & $37,437.981$ & $-88,527$ \\
\hline & $(0.049)$ & $(0.037)$ & $(0.130)$ & & $(0.010)$ & (1358) & \\
\hline \multirow[t]{2}{*}{ Model 4} & 0.306 & 0.081 & -0.640 & 0.044 & 0.032 & $37,216.324$ & $-89,431^{a}$ \\
\hline & $(0.048)$ & $(0.035)$ & $(0.141)$ & $(0.016)$ & $(0.012)$ & (1168) & \\
\hline
\end{tabular}

$\mathrm{h}^{2}=$ heritability for the direct genetic effect; $\mathrm{m}^{2}=$ heritability for the maternal genetic effect; $\mathrm{c}^{2}=$ estimate for the ratio of permanent environmental variance to phenotypic variance; $r_{a m}=$ correlation between additive genetic effect and maternal genetic effect; $1^{2}=$ estimate for the ratio of cytoplasmic line variance to phenotypic variance; $\sigma^{2}=$ phenotypic variance; logCPO = average logarithm of the conditional predictive ordinate.

a Model with the best fit with observations.

aBrW-mADG using Models 6 and 8, suggesting that genetic correlation estimated for these two genetic effects may be spurious. The inclusion of the $\mathbf{l}$ effect increased the direct genetic correlation estimated between the pair aBrW-aWW while correlation between the pairs aBrW-aADG and aWWaADG decreased. In any case, the latter genetic correlation is still over 0.9 or higher. Also, the inclusion of the $\mathbf{1}$ effect in the estimation models gave a substantial increase of the correlation between the maternal genetic effects associated with birth weight (pairs mBrW-mWW and mBrW-mADG) while correlation for the pair mWW-mADG remained substantially the same. It is important to note that genetic correlations for the pairs involving the maternal genetic effect for BrW were the parameters with the highest modification when Model 6 was fitted. This suggests that estimates obtained under this model are the most reliable for them. Genetic correlations among direct and maternal genetic effects across traits greatly changed (with respect to Model 5) tending to increase when using Model 7 but without particular pattern when using Model 8. Correlations estimated for the pairs aWW-aADG and mWW-mADG suggest that the genes involved in regulation of these pairs of genetic effects are the same.

Table 5 gives the correlations between either $\mathbf{c}$ or $\mathbf{l}$ effects associated with each of the analysed traits. Most correlations associated with BrW had large SD's and those for the pairs cBrW-cWW and lBrW-lWW were not significant. However, correlations between the pairs cWW-cADG (over 0.9) and
IWW-lADG (roughly 1) were positive and very high showing that both maternal permanent environmental and cytoplasmic line effects associated with these traits are basically the same.

\section{Discussion}

In this paper we present new estimates of the influence of the cytoplasmic line effect on genetic parameters associated with preweaning growth traits in beef cattle. Analyses were carried out, for the first time in this breed, using a Bayesian methodology that is still rarely applied in these livestock (Yagüe et al., 2009).

Genetic parameters estimated here are basically consistent with others previously estimated for preweaning growth traits using the CORECA database and REML estimation procedures. Gutiérrez et al. (2007), using an estimation model comparable with current Model 5, reported estimates of $\mathrm{h}^{2}$ and $\mathrm{m}^{2}$ of $0.390 \pm 0.030$ and $0.208 \pm 0.020$, respectively for $\mathrm{BrW}$, and $0.429 \pm 0.012$ and $0.112 \pm 0.003$ for WW. In general, whatever the estimation model used, estimates of $\mathrm{h}^{2}, \mathrm{~m}^{2}$ and $r_{a m}$ obtained in the current study for BrW are slightly higher ( $r_{a m}$ more negative) than those recently reported by Gutiérrez et al. (2006, 2007) but clearly lower than others previously estimated using poorer structured databases (Goyache et al., 2003a; Gutiérrez et al., 1997). However, this is not true for WW and ADG when multivariate estimation models included the $\mathbf{l}$ effect. In these cases, estimates of $\mathrm{h}^{2}$ 
Table 3

Mean and SD (in brackets) of the posterior distribution for the within-trait genetic and environmental parameters included in the four multivariate models fitted.

\begin{tabular}{|c|c|c|c|c|c|c|}
\hline & $\mathrm{h}^{2}$ & $\mathrm{~m}^{2}$ & $r_{a m}$ & $c^{2}$ & $1^{2}$ & $\sigma^{2}$ \\
\hline \multicolumn{7}{|c|}{ Birth weight } \\
\hline \multirow[t]{2}{*}{ Model $5^{\mathrm{a}}$} & 0.396 & 0.285 & -0.370 & & & 39.663 \\
\hline & $(0.032)$ & $(0.015)$ & $(0.032)$ & & & $(0.809)$ \\
\hline \multirow[t]{2}{*}{ Model $6^{\mathrm{b}}$} & 0.412 & 0.106 & -0.257 & 0.088 & & 42.261 \\
\hline & $(0.031)$ & $(0.032)$ & $(0.067)$ & $(0.011)$ & & $(1.053)$ \\
\hline \multirow[t]{2}{*}{ Model $7^{c}$} & 0.544 & 0.374 & -0.513 & & 0.007 & 37.478 \\
\hline & $(0.035)$ & $(0.038)$ & $(0.049)$ & & $(0.003)$ & (1.139) \\
\hline \multirow[t]{2}{*}{ Model $8^{\mathrm{d}}$} & 0.436 & 0.290 & -0.490 & 0.037 & 0.016 & 38.206 \\
\hline & $(0.014)$ & $(0.005)$ & $(0.014)$ & $(0.007)$ & $(0.004)$ & $(0.627)$ \\
\hline \multicolumn{7}{|c|}{ Weaning weight } \\
\hline \multirow[t]{2}{*}{ Model 5} & 0.429 & 0.112 & -0.282 & & & 1488.085 \\
\hline & $(0.012)$ & $(0.002)$ & $(0.016)$ & & & (15.589) \\
\hline \multirow[t]{2}{*}{ Model 6} & 0.397 & 0.127 & -0.472 & 0.039 & & 1387.559 \\
\hline & $(0.021)$ & $(0.003)$ & $(0.033)$ & $(0.007)$ & & (18.307) \\
\hline \multirow[t]{2}{*}{ Model 7} & 0.309 & 0.126 & -0.371 & & 0.030 & 1431.339 \\
\hline & $(0.013)$ & $(0.003)$ & $(0.014)$ & & $(0.006)$ & $(14.537)$ \\
\hline \multirow[t]{2}{*}{ Model 8} & 0.211 & 0.069 & -0.173 & 0.023 & 0.029 & 1460.117 \\
\hline & $(0.003)$ & $(0.001)$ & $(0.007)$ & $(0.004)$ & $(0.004)$ & (13.469) \\
\hline \multicolumn{7}{|c|}{ Average daily gain } \\
\hline \multirow[t]{2}{*}{ Model 5} & 0.307 & 0.104 & -0.270 & & & $39,896.861$ \\
\hline & $(0.009)$ & $(0.003)$ & $(0.024)$ & & & $(424)$ \\
\hline \multirow[t]{2}{*}{ Model 6} & 0.284 & 0.126 & -0.485 & 0.033 & & $37,638.111$ \\
\hline & $(0.008)$ & $(0.004)$ & $(0.038)$ & $(0.007)$ & & $(547)$ \\
\hline \multirow[t]{2}{*}{ Model 7} & 0.234 & 0.108 & -0.416 & & 0.031 & $38,423.656$ \\
\hline & $(0.019)$ & $(0.003)$ & $(0.022)$ & & $(0.006)$ & (389) \\
\hline \multirow[t]{2}{*}{ Model 8} & 0.169 & 0.058 & -0.326 & 0.037 & 0.031 & $38,663.468$ \\
\hline & $(0.002)$ & $(0.001)$ & $(0.006)$ & $(0.007)$ & $(0.005)$ & (378) \\
\hline
\end{tabular}

$\mathrm{h}^{2}=$ heritability for the direct genetic effect; $\mathrm{m}^{2}=$ heritability for the maternal genetic effect; $c^{2}=$ estimate for the ratio of permanent environmental variance to phenotypic variance; $r_{a m}=$ correlation between additive genetic effect and maternal genetic effect; $\mathrm{l}^{2}=$ estimate for the ratio of cytoplasmic line variance to phenotypic variance; = phenotypic variance; $\log \mathrm{CPO}=$ average logarithm of the conditional predictive ordinate.

a $\log \mathrm{CPO}=-241,053$.

b $\log \mathrm{CPO}=-245,899$.

c $\log \mathrm{CPO}=-241,108$.

d $\log \mathrm{CPO}=-232,804$.

and $\mathrm{m}^{2}$ for WW and ADG tend to be lower and $\mathrm{r}_{\mathrm{am}}$ less negative than those previously estimated by Gutiérrez et al. (2006, 2007). The current scenario is more consistent with most estimates for WW and ADG available in the literature (see Koots et al., 1994a, 1994b; Gutiérrez et al., 2007 for reviews). Therefore, this suggests that: a) at least for WW and ADG, some major environmental factors were not included in previous estimation models causing an inflation of the estimates of the genetic effects; and b) these previously unaccounted environmental factors may be partially linked to cytoplasmic line.

In any case, the effect of cytoplasmic line on the assessed traits is low. Moreover, the influence of the $\mathbf{l}$ effect on BrW may be null. This is consistent with earlier reports on this issue in beef cattle (Gibson et al., 1997; Mezzadra et al., 2005; Rohrer et al., 1994; Tess and MacNeil, 1994; Tess et al., 1987). The low effect assessed for cytoplasmic line may be partially due to its maternal inheritance. Selection intensity in females is much lower than in males, and females give much fewer progeny. Also, most published analyses in sheep failed in finding any important effect of cytoplasmic line for

\section{Table 4}

Mean and SD (in brackets) of the posterior distribution for the correlations between either genetic effects for all the analyzed traits.

\begin{tabular}{|c|c|c|c|c|}
\hline Correlation & Model 5 & Model 6 & Model 7 & Model 8 \\
\hline \multirow[t]{2}{*}{ aBrW-aWW } & 0.486 & 0.424 & 0.552 & 0.550 \\
\hline & $(0.022)$ & $(0.037)$ & $(0.038)$ & $(0.010)$ \\
\hline \multirow[t]{2}{*}{ aBrW-aADG } & 0.399 & 0.308 & 0.352 & 0.319 \\
\hline & $(0.034)$ & $(0.024)$ & $(0.028)$ & (0.009) \\
\hline \multirow[t]{2}{*}{ aWW-aADG } & 0.977 & 0.932 & 0.912 & 0.877 \\
\hline & $(0.006)$ & $(0.006)$ & $(0.007)$ & $(0.002)$ \\
\hline \multirow[t]{2}{*}{$\mathrm{mBrW}-\mathrm{mWW}$} & 0.391 & 0.087 & 0.459 & 0.453 \\
\hline & $(0.015)$ & $(0.028)$ & $(0.027)$ & $(0.008)$ \\
\hline \multirow[t]{2}{*}{ mBrW-mADG } & 0.202 & -0.117 & 0.227 & 0.225 \\
\hline & $(0.015)$ & $(0.020)$ & $(0.037)$ & $(0.009)$ \\
\hline \multirow[t]{2}{*}{$\mathrm{mWW}-\mathrm{mADG}$} & 0.973 & 0.940 & 0.955 & 0.899 \\
\hline & $(0.002)$ & $(0.003)$ & $(0.003)$ & $(0.001)$ \\
\hline \multirow[t]{2}{*}{ aBrW-mWW } & -0.137 & -0.054 & -0.236 & -0.051 \\
\hline & $(0.026)$ & $(0.027)$ & $(0.012)$ & (0.009) \\
\hline \multirow[t]{2}{*}{$\mathrm{aBrW}-\mathrm{mADG}$} & -0.019 & 0.049 & -0.162 & 0.103 \\
\hline & $(0.023)$ & $(0.024)$ & $(0.015)$ & (0.009) \\
\hline \multirow[t]{2}{*}{ aWW-mBrW } & -0.222 & -0.148 & -0.317 & -0.379 \\
\hline & $(0.021)$ & $(0.040)$ & $(0.018)$ & $(0.007)$ \\
\hline \multirow[t]{2}{*}{ aWW-mADG } & -0.293 & -0.420 & -0.389 & -0.171 \\
\hline & $(0.022)$ & $(0.024)$ & $(0.023)$ & $(0.007)$ \\
\hline \multirow[t]{2}{*}{ aADG-mBrW } & -0.203 & -0.031 & -0.134 & -0.212 \\
\hline & $(0.025)$ & $(0.026)$ & $(0.024)$ & $(0.007)$ \\
\hline \multirow{2}{*}{ aADG-mWW } & -0.234 & -0.416 & -0.308 & -0.122 \\
\hline & $(0.018)$ & $(0.049)$ & $(0.010)$ & $(0.007)$ \\
\hline
\end{tabular}

aBrW, aWW and aADG are, respectively, the direct animal genetic effects for Birth Weight, Weaning Weight and Average Daily Gain; mBrW, mWW and mADG are, respectively, the maternal genetic effects for Birth Weight, Weaning Weight and Average Daily Gain.

birth or weaning weight (Hanford et al., 2003; Snowder et al., 2004; Van Vleck et al., 2002). However, unlike weaning weight, the inclusion of the cytoplasmic line effect in the models fitted for the analyses of birth weight in sheep did not improve the model.

The present study clearly shows the difficulties in identifying cytoplasmic line effects using performance recording databases. In univariate analyses the full model, including both the $\mathbf{c}$ and the $\mathbf{1}$ effect, tended to have the better fit with data. However, this was not true for the multivariate

Table 5

Mean and SD (in brackets) of the posterior distribution for the correlations between either permanent environmental effects associated with dam (c) and either cytoplasmic line (1) effects.

\begin{tabular}{lllr}
\hline $\begin{array}{l}\text { Permanent material } \\
\text { environment effect }\end{array}$ & cWW & \multicolumn{1}{l}{ cADG } \\
\hline Model 6 & cBrW & $0.560(0.097)$ & $0.216(0.109)$ \\
& cWW & & $0.902(0.035)$ \\
Model 8 & cBrW & $0.191(0.111)$ & $-0.273(0.078)$ \\
& cWW & & $0.885(0.038)$ \\
\multicolumn{2}{l}{ Cytoplasmic line effect } & IWW & lADG \\
\hline Model 7 & lBrW & $-0.462(0.205)$ & $-0.599(0.197)$ \\
& lWW & & $0.980(0.012)$ \\
Model 8 & lBrW & $-0.324(0.203)$ & $-0.478(0.185)$ \\
& lWW & & $0.979(0.013)$
\end{tabular}

cBrW, cWW, and cADG are, respectively, the environmental effect associated with dam for Birth Weight, Weaning Weight and Average Daily Gain; BrW, IWW, and IADG are, respectively, the cytoplasmic line effect for Birth Weight, Weaning Weight and Average Daily Gain. 
analyses. In these cases, $\log \mathrm{CPO}$ suggests that the best fit with data was for Model 6 which did not include the 1 effect. However, the SD of the estimates of correlations between genetic effects given in Table 4 for Model 7 (which only included the $\mathbf{l}$ effect) are lower than those estimated for Model 6, which included the $\mathbf{c}$ effect. Estimates different from zero give additional information. Excluding the pairs aWW-aADG and mWW-mADG, coefficients of variation of the estimates obtained using Model 7 varied from 3\% to $18 \%$ while those estimated using Model 6 varied from 6\% to 84\%.

Overall, we can suggest that, despite difficulties in estimation, the $\mathbf{l}$ effect is not negligible. Looking at the univariate results, it must be noted that different conclusions could be obtained if criteria to choose a model are based on the significance of the parameters or by $\log \mathrm{CPO}$. A very detailed data structure is needed to separate cytoplasmic and permanent environment effects, the latter being nested in the former from the maternal genetic effect.

To account for the non genetic relationship between maternal effects in adjacent generations, Quintanilla et al. (1999) included in their estimation model a matrix $\mathbf{E}$ including the correlation between maternal environments provided by a dam and its daughters. As reported by Quintanilla et al. (1999), this approach lead to less biased estimates of genetic effects and direct and maternal breeding values. Conceptually, this more sophisticated approach accounts properly for the dispersion structure of data even when the maternal pedigree is incomplete, by introducing phantom dams for animals with records but unknown dam. However, the Quintanilla et al.'s (1999) E matrix is equivalent to the correlation matrix between cytoplasmatic effects of mitochondrial DNA proposed by Kennedy and Schaeffer (1990) if the alteration of mitochondrial DNA occurs at a constant rate from generation to generation.

If 1 effects are basically determined by mitochondrial DNA, it would be difficult to discriminate between it and the maternal environmental effects, given the high similarity between the incidence and dispersion matrices of these effects (Gibson et al., 1997; Quintanilla et al., 1999). Correct estimates of $\mathbf{l}$ effects could be possible when maternal lineages are sufficiently deep (Salehi and James, 1997) and performance of a number of representatives of each line has been recorded (Gibson et al., 1997). In our case, the average number of cows producing data per line was about 3, with a mean number of records per line of 7 . Therefore, the deepness of the maternal lineages available here could be limiting the estimation of the $\mathbf{l}$ effects. However, other studies such as that by Roughsedge et al (2000) in dairy cattle carried out similar analyses using maternal lines containing 5 or more cows. Potential sources of bias and reduction of the power of estimation of cytoplasmic inheritance are incomplete and incorrect pedigree information being used in the assignment of maternal lineages. Using simulations in dairy cattle, Roughsedge et al. (2001) reported that a pedigree error rate of $8 \%$ per generation would result in a $75 \%$ reduction in the estimable magnitude of a $5 \%$ true $\mathbf{l}$ component of variance after nine generations. However, contrary to that found in the current study, if this were the sole cause of these low estimates, the use of more information using multivariate models would by itself improve the quality of the estimations.

Even in scenarios of complete and deep pedigrees it is possible that some cytoplasmic lines share mitochondrial
DNA sequences by unaccounted ancient ancestry or simply by state. Molecular identification of mitochondrial DNA variants could overcome these problems directly assigning individuals to cytoplasmic lineage groups. However, using this approach can also fail in identifying $\mathbf{l}$ effects on performance (Rohrer et al., 1994). It is not easy to ascertain if mitochondrial DNA variants, usually identified on the hypervariable control region of mitochondrial DNA sequences, are representative of the mitochondrial gene variants influencing performance. Also, there is evidence of the existence of autosomal genes involved in moderating mitochondrial activity (Clempson et al., 2011). These genes may vary among the member of a given cytoplasmic line or group, therefore affecting the estimation of the $\mathbf{l}$ component.

In summary, current results agree with previous reports suggesting that cytoplasmic line may have a marginal effect on growth performance in beef cattle. Also, the inclusion of the $\mathbf{l}$ effect on estimation models does not improve the fitness with data. Therefore, cytoplasmic line may have a marginal effect on growth performance in beef cattle but not sufficient in magnitude to justify the inclusion of the $\mathbf{l}$ effect in models in beef improvement schemes. Despite the facts summarized above, environmental covariances between the maternal effects shown by a dam and its daughters, probably linked to mitochondrial inheritance, are likely to exist (Quintanilla et al., 1999). Difficulties in correctly assessing the importance of this kind of effect may be more likely to be linked to a poor quality or structure of the filed data available for genetic studies rather than a negligible effect.

\section{Acknowledgements}

This paper was partially funded by a grant from CICYTFEDER, No. 1FD97-1633, and the Regional Government of Principado de Asturias through the Consejería de Medio Rural y Pesca. The authors would like to thank the staff at Área de Genética y Reproducción Animal of SERIDA and especially Teresa Geijo for their kind support and the editing of our database.

\section{References}

Cervantes, I., Gutiérrez, J.P., Fernández, I., Goyache, F., 2010. Genetic relationships among calving ease, gestation length, and calf survival at weaning in the Asturiana de los Valles beef cattle breed. J. Anim. Sci. 88, 96-101.

Clempson, A.M., Pollott, G.E., Brickell, J.S., Bourne, N.E., Munce, N., Wathes, D. C., 2011. Polymorphisms in the autosomal genes for mitochondrial function TFAM and UCP2 are associated with performance and longevity in dairy cows. Animal 5 (9), 1335-1343.

Garmyn, A.J., Moser, D.W., Christmas, R.A., Minick Bormann, J., 2010. Estimation of genetic parameters and effects of cytoplasmic line on scrotal circumference and semen quality traits in Angus bulls. J. Anim. Sci. 89 (3), 693-698.

Gibson, J.P., Freeman, A.E., Boettcher, P.J., 1997. Cytoplasmic and mitochondrial inheritance of economic traits in cattle. Livest. Prod. Sci. 47, 115-124.

Goyache, F., Fernández, I., Royo, L.J., Álvarez, I., Gutiérrez, J.P., 2003a. Factors affecting actual weaning weight, preweaning average daily gain and relative growth rate in Asturiana de los Valles beef cattle breed. Arch. Anim. Breed. 46, 235-244.

Goyache, F., Gutiérrez, J.P., 2001. Heritability of reproductive traits in Asturiana de los Valles beef cattle breed. Arch. Tierzücht. 44, 489-496.

Goyache, F., Gutiérrez, J.P., Álvarez, I., Fernández, I., Royo, L.J., Gomez, E., 2003b. Genetic analysis of calf survival at different preweaning ages in beef cattle. Livest. Prod. Sci. 83, 13-20. 
Goyache, F., Gutiérrez, J.P., Fernández, I., Royo, L.J., Álvarez, I., 2005. Genetic analysis of days open in beef cattle. Livest. Prod. Sci. 93, 283-289.

Gutiérrez, J.P., Cañón, J., Goyache, F., 1997. Estimation of direct and maternal genetic parameters for preweaning traits in the Asturiana de los Valles beef cattle breed through animal and sire models. J. Anim. Breed. Genet. 114, 261-266.

Gutiérrez, J.P., Fernández, I., Álvarez, I., Royo, L.J., Goyache, F., 2006. Sire × contemporary group interactions for birth weight and preweaning growth traits in the Asturiana de los Valles beef cattle breed. Livest. Sci. 99, 61-68.

Gutiérrez, J.P., Goyache, F., 2002. Estimation of genetic parameters of type traits in beef cattle. J. Anim. Breed. Genet. 119, 93-100.

Gutiérrez, J.P., Goyache, F., Fernández, I., Alvarez, I., Royo, L.J., 2007. Genetic relationships among calving ease, calving interval, birth weight, and weaning weight in the Asturiana de los Valles beef cattle breed1. J. Anim. Sci. 85, 69-75.

Hanford, K.J., Snowder, G.D., Van Vleck, L.D., 2003. Models with nuclear, cytoplasmic, and environmental effects for production traits of Columbia sheep. J. Anim. Sci. 81, 1926-1932.

Kennedy, B.W., Schaeffer, L.R., 1990. Reproductive technology and genetic evaluation. In: Gianola, D., Hammond, K. (Eds.), Advances in Statistical Methods for Genetic Improvement of Livestock. Springer-Verlag, New York, pp. 507-532.

Koots, K.R., Gibson, J.P., Smith, C., Wilton, J.W., 1994a. Analyses of published genetic parameter estimates for beef production traits. 1. Heritability. Anim. Breed. Abstr. 62, 309-338.

Koots, K.R., Gibson, J.P., Smith, C., Wilton, J.W., 1994b. Analyses of published genetic parameter estimates for beef production traits. 2. Phenotypic and genetic correlations. Anim. Breed. Abstr. 62, 825-853.

Legarra, A., 2008. TM Threshold Model. http://acteon.webs.upv.es/.

Legates, J.E., 1972. The role of maternal effects in animal breeding: IV. Maternal effects in laboratory species. J. Anim. Sci. 35, 1294-1302.

Maniatis, N., Pollott, G.E., 2002. Nuclear, cytoplasmic, and environmental effects on growth, fat, and muscle traits in Suffolk lambs from a sire referencing scheme. J. Anim. Sci. 80, 57-67.

Mezzadra, C., Melucci, L.M., CorvaI, P.M., López, S., Rípoli, M.V., Lirón, P., Giovambattista, G., 2005. Effects of cytoplasmic inheritance on preweaning traits of Hereford cattle. Genet. Mol. Biol. 28 (3), 357-362.
Northcutt, S.L., Willham, R.L., Wilson, D.E., 1991. Genetic parameters for nuclear and nonnuclear inheritance in three synthetic lines of beef cattle differing in mature size. J. Anim. Sci. 69, 4745-4753.

Pettit, L.I., Young, K.D.S., 1990. Measuring the effect of observations on Bayes factors. Biometrika 77, 455-466.

Quintanilla, R., Varona, L., Pujol, M.R., Piedrahita, J., 1999. Maternal animal model with correlation between maternal environmental effects of related dams. J. Anim. Sci. 77, 2904-2917.

Rohrer, G.A., Taylor, J.F., Sanders, J.O., Thallman, R.M., 1994. Evaluation of line and breed of cytoplasm effects on performance of purebred Brangus cattle. J. Anim. Sci. 72, 2798-2803.

Roughsedge, T., Visscher, P.M., Brotherstone, S., 2000. Effects of cow families on production traits in dairy cattle. J. Anim. Sci. 71, 49-57.

Roughsedge, T., Brotherstone, S., Visscher, P.M., 2001. Bias and power in the estimation of a maternal family variance component in the presence of incomplete and incorrect pedigree information. J. Dairy Sci. 84, 944-950.

Salehi, A., James, J.W., 1997. Detection of cytoplasmic effects on production: the influence of number of years of data. Genet. Sel. Evol. 29, 269-277.

Snowder, G.D., Hanford, K.J., Van Vleck, L.D., 2004. Comparison of models including cytoplasmic effects for traits of Rambouillet sheep. Livest. Prod. Sci. 90, 159-166.

Tess, M.W., MacNeil, M.D., 1994. Evaluation of cytoplasmic genetic effects in Miles City line 1 Hereford cattle. J. Anim. Sci. 72, 851-856.

Tess, M.W., Reodacha, C., Robison, O.W., 1987. Cytoplasmic genetic effects on preweaning growth and milk yield in Hereford cattle. J. Anim. Sci. 65, 675-684.

Tess, M.W., Robison, O.W., 1990. Evaluation of cytoplasmic genetic effects in beef cattle using an animal model. J. Anim. Sci. 68, 1899-1909.

Van Vleck, L.D., Snowder, G.D., Hanford, K.J., 2002. Models with cytoplasmic effects for birth, weaning and fleece weights, and litter size at birth for a population of Targhee sheep. J. Anim. Sci. 81, 61-67.

Varona, L., Moreno, C., Garcia-Cortes, L.A., Altarriba, J., 1997. Model determination in a case of heterogeneity of variance using sampling techniques. J. Anim. Breed. Genet. 114, 1-12.

Varona, L., Sorensen, L., 2010. A genetic analysis of mortality in pigs. Genetics 184 (1), 277-284.

Yagüe, G., Goyache, F., Becerra, J., Moreno, C., Sánchez, L., Altarriba, J., 2009. Bayesian estimates of genetic parameters for pre-conception traits, gestation length and calving interval in beef cattle. Anim. Reprod. Sci. 114, 72-80. 